\title{
Highly efficient and durable antibacterial cotton fabrics finished with zwitterionic polysulfobetaine by one-step eco- friendly strategy
}

\author{
Simeng Gao $\cdot$ Jing Su $\cdot$ Wencong Wang $\cdot$ Jiajia Fu $\cdot$ Hongbo Wang $\mathbb{D}$
}

Received: 24 March 2020/Accepted: 15 October 2020/Published online: 10 November 2020

(C) Springer Nature B.V. 2020

\begin{abstract}
In this work, a novel formulation of polysulfobetaine, poly (sulfobetaine-acrylamide-allyl glycidyl ether) (PSPB-AM-AGE), was synthesized and grafted onto cotton. The synthesis of PSPB-AMAGE and its grafting on the cotton fabrics were confirmed by FTIR, XPS and SEM. The PSPB-AMAGE treated cotton fabrics exhibited a high level of antibacterial rate against both Escherichia coli (E. coli) and Staphylococcus aureus (S. aureus), which are $95.18 \%$ and $98.74 \%$, separately, as well as a good laundry durability. The mechanical tests showed that the essential cotton properties can be largely preserved in the treatment process. Moreover,
\end{abstract}

Electronic supplementary material The online version of this article (https://doi.org/10.1007/s10570-020-03542-7) contains supplementary material, which is available to authorized users.

S. Gao · J. Su $(\bowtie) \cdot$ W. Wang $\cdot$ J. Fu $\cdot$ H. Wang $(\bowtie)$ Jiangsu Engineering Technology Research Centre of Functional Textiles, Jiangnan University, Wuxi, China e-mail: sujing@jiangnan.edu.cn

H. Wang

e-mail: wxwanghb@jiangnan.edu.cn

S. Gao

e-mail: jndxgaosm@163.com

W. Wang

e-mail: wencong828@hotmail.com

S. Gao $\cdot$ J. Su $\cdot$ W. Wang $\cdot$ J. Fu $\cdot$ H. Wang

Key Laboratory of Eco-Textiles, Ministry of Education, Jiangnan University, Wuxi, China the hydrophilicity, air and water permeability of the cotton were improved after treated with PSPB-AMAGE, indicating a better wearing comfort performance. The whiteness of the cotton fabrics did not decrease significantly. The safety evaluation demonstrated that PSPB-AM-AGE had no cytotoxicity. The developed antibacterial finishing introduced a new method to apply polysulfobetaine interfaced on cellulose, providing great potential for biomedical fabric application.

Keywords Betaine - Polymerization - Cotton fabric · Antibacterial finishing

\section{Introduction}

Cotton fabrics have been widely used in modern societies as a natural fiber due to their comfort, breathability, and biocompatibility. However, it can absorb secretions of skin (Chen 2016) owing to the porous structure and good moisture absorption (Zhang 2018), which provides suitable conditions such as nutrient sources, appropriate temperature, and humidity for the growth of microorganisms (Fei 2018; Lu 2019). The rapid growth of these microorganisms can be extremely harmful to public health, since pathogenic bacterial lead to potential cross-linking infections (He 2017) (Si 2018) (Hanczvikkel 2019) (Mitra 
2019). Therefore, to prevent bacterial infections of the human body, it is of great importance to developing antibacterial cotton fabrics.

Antibacterial finishing agents play a pivotal role in constructing antibacterial textile surfaces, including inorganic, organic, and natural antibacterial agents (Fei 2018). The widely used inorganic antibacterial agents in the literature (Gao 2019; Rehan 2018) are Ag and $\mathrm{Cu}$ nanoparticles, with broad-spectrum and highefficiency antibacterial characteristics. Recently, usage of polymeric binder has been focused to enhance the adhesion between the cotton and nanoparticles (El-Nahhal 2020) (Shen, William 2019).Whereas, a problem revealed with these nanoparticles is that nanoscale metals leached into the environment can cause damage such as cytotoxicity and genotoxicity to organisms (Ding 2018; Peddinti 2019) The chitosan as the representative of natural antibacterial agents tends to be eco-friendly, which is abundant in the earth. However, the applications of the chitosan are restricted due to the $\mathrm{pH}$ sensitive of finishing solution (Shahid Islam 2020) and poor adhesion to cotton fibers without the cross-linker (Lim 2003). Plenty of organic agents such as quaternary ammonium compounds (QACs) (Lu 2019), N-halamine (Liu 2017; Wang 2018), triclosan, and zwitterionic materials are introduced to impart biocidal functions (Hassan 2017). Questions have been raised about the drug resistance of the prolonged use of QACs and triclosan (Chen 2011a; Wu 2015). Although N-halamine and triclosan have high antibacterial efficacy (Li 2019), they are highly toxic and have negative effects on the human body (Dann 2011; Wang 2018). In addition, N-halamine can damage the mechanical properties of cotton fibers (Li 2019). Recently, zwitterionic materials, especially betaines, have been reported for the characteristics such as antimicrobial activity, antimicrobial adhesion (Zhang 2018), antifouling (Zhang 2006), nonirritant to the skin, nontoxic to animals (Chang 2011; Chen 2011a). It has received great attention in the field of biological materials, biosensor, environmental engineering (Chen 2011b; Li 2012; Sivashanmugan 2017). Betaines, therefore, may present a promising opportunity to develop eco-friendly and effective antimicrobial agents.

For decades, studies have documented the issues of betaine groups, molecular weight, and the ratio of hydrophobic to hydrophilic sections are considerable for the antimicrobial activities (Ganewatta 2015; Ward 2006; Wieczorek 2017). Ward et al. demonstrated the minimum inhibitory concentration (MIC) values of polysulfobetaine agents for $S$. aureus and E. coli are in the range of $1125-2000 \mu \mathrm{g} / \mathrm{mL}$. They also found that microbiocidal effects are related to compositions of the copolymer (Ward 2006). Others also focused on how to construct an antimicrobial surface coating with betaine and its derivatives ( $\mathrm{Li}$ 2012; Sundaram 2014). Li et al. modified silicone with polysulfobetaine chains and poly (ethylene glycol) dimethacrylate, which was served as the anchor for the attachment of polysulfobetaine, resulting in antiprotein and anti-platelet adhesion and good blood compatibility (Li 2012). However, their application in the design of the antibacterial cotton textile surface is seldom so far.

Chen et al. synthesized sulfopropylbetaine with alkoxysilane groups or isocyanate as antibacterial finishing agents (AFA) to react with hydroxyl groups on cotton fabrics (Chen 2011a; Chen 2016). For another, a sulfobetaine containing triazine groups was designed by $\mathrm{He}$ et al. and grafted onto cellulosic fabrics (He 2016). However, the textiles grafted with these agents exhibited poor durability, since the antibacterial agents they used are small molecular substances, which would show a higher level of MIC against microbes and prone to leach into the environment due to their high solubility in water. There had been no systematic study on the control of polysulfobetaine on cotton textiles to form a robust antimicrobial surface. Our previous work indicated that sulfopropylbetaine copolymers bearing carboxylic groups can provide a durable grafting layer on cellulose fibers with improved microbicide potency (Zhou 2018). Since the pH of AFA is low and the temperature of the finishing process is high, there is an obvious decline in the breaking strength of finished textiles, thus could not meet the required mechanical property.

In this work, we report a novel approach for covalently grafting sulfopropylbetaine copolymers onto cotton textiles through a ring opening reaction of epoxy and hydroxyl groups. The monomer of primary interest is a zwitterionic sulfopropylbetaine (SPB) that served as a microbiocidal and antibacterial adhesion group. For covalent anchoring on the cotton fabrics, allyl glycidyl ether (AGE), highly reactive with nucleophiles via nucleophilic reaction, was 
selected as a functional group. To avoid gelation, we introduced acrylamide (AM) into the copolymers as a link group in the polymerization. The long hydrocarbon chain the copolymers will increase the hydrophobic and the microbiocidal effects. Then, the poly (sulfopropylbetaine-acrylamide-allyl glycidyl ether) (PSPB-AM-AGE) is synthesized, characterized, and used for AFA. After the grafting of PSPB-AM-AGE to the cotton substrates, the in vitro antibacterial activity, mechanical properties, water absorption behavior, air/moisture permeability of fabric treated with PSPB-AM-AGE and raw cotton are systematically assessed.

\section{Experimental section}

Materials

1,3-Propanesultone (1,3-PS, 99\%), N, N-dimethyallylamine (DMAA, 98\%), tetrahydrofuran (THF, AR), and ammonium persulfate (APS) were purchased from Sinopharm Chemical Reagent Co., Ltd. Acrylamide (AM) and allyl glycidyl ether (AGE) were obtained from Aladdin Reagent Company. All chemicals were used as received. Gram-positive bacteria ( $S$. aureus, ATCC6538) and gram-negative bacteria (E. coli, ATCC8739) were from Shanghai Xiejiu Bio-Tech Co., Ltd. HaCat cells purchased from iCell Bioscience Inc, Shanghai. Cotton fabric characteristics: combed plain woven cotton fabric (yarn with a linear density of 14.5 tex; warp and weft density of fabrics are 524 and 283 pieces/ $(10 \mathrm{~cm})$, respectively), from Huafang Co., Ltd (China).

\section{Synthesis of SPB, PSPB-AM-AGE}

SPB was synthesized in a similar fashion as the procedure of our previous work (Zhou 2018). Briefly, 1,3-propanesultone (5.99 g, $0.049 \mathrm{~mol}$ ) was dissolved in $50 \mathrm{~mL}$ THF under stirring at $500 \mathrm{r} / \mathrm{min}$. This solution was heated to $60{ }^{\circ} \mathrm{C}$ and maintained at the temperature throughout the reaction period. An oxygen-free environment was then established in the system by degassing with nitrogen. Nitrogen is bubbled through the mixture of another $20 \mathrm{~min}$. DMAA $(5.02 \mathrm{~g}, 0.059 \mathrm{~mol}$, dissolved in $60 \mathrm{~mL}$ THF) was then added dropwise to the mixture using a syringe over a $60 \mathrm{~min}$ period. The reaction was allowed to proceed under a nitrogen atmosphere with stirring for $6 \mathrm{~h}$. The resulting product SPB was collected through rotary evaporation.

Then, various amounts of SPB, AM, and AGE monomers were firstly added into distilled water to form a $1 \mathrm{M}$ solution and then were stirred for $10 \mathrm{~min}$. The mixed solution was then purged with nitrogen for $20 \mathrm{~min} .1 \%$ eq. of APS was then added into the solution, and it was mixed for another $20 \mathrm{~min}$. The solution reacted at $70{ }^{\circ} \mathrm{C}$ for $4 \mathrm{~h}$ and the reaction was terminated at room temperature. The products were precipitated in acetone and purified by ethyl alcohol three times. Afterward, the product was freeze dried using a lyophilizer, and a white crystal powder was obtained. The synthetic route of PSPB-AM-AGE is shown in Scheme 1.

\section{Antibacterial finishing of cotton fabrics}

A piece of cotton fabric $(5 \times 10 \mathrm{~cm})$ was cleaned with DI water to remove surface impurities by ultrasonication and dried in air. Thereafter, PSPB-AMAGE was dissolved in water at concentrations of $9 \%$. Cotton fabrics were dipped in the PSPB-AM-AGE solution for $60 \mathrm{~min}$ at $60{ }^{\circ} \mathrm{C}$ The route of two dips and two nips was applied to control the wet condition of textiles. Controlling the wet pick of textiles as $60 \%$ of its pristine weight by controlling pressure during padding. Then PSPB-AM-AGE was firmly immobilized on cotton fibers by dried at $70{ }^{\circ} \mathrm{C}$ for $5 \mathrm{~min}$ and cured at $165{ }^{\circ} \mathrm{C}$ for $3 \mathrm{~min}$. Samples were washed with water and dried at ambient temperature. After finishing, the weight of cotton fabrics approximately had increased by $5 \%$.

\section{Characterization}

The chemical structure of the PSPB-AM-AGE copolymer was characterized by FTIR, ${ }^{1} \mathrm{H}$ NMR spectra and GPC. ATR-FTIR spectra of the pristine and PSPBAM-AGE treated cotton fabric were obtained using a Nicolet iS10. Elemental analyses were investigated by X-ray photoelectron spectroscopy (Thermo Scientific ESCALAB, USA). The surface morphologies of samples were observed by scanning electron microscopy (SEM) images (Hitachi SU1510, Japan). 


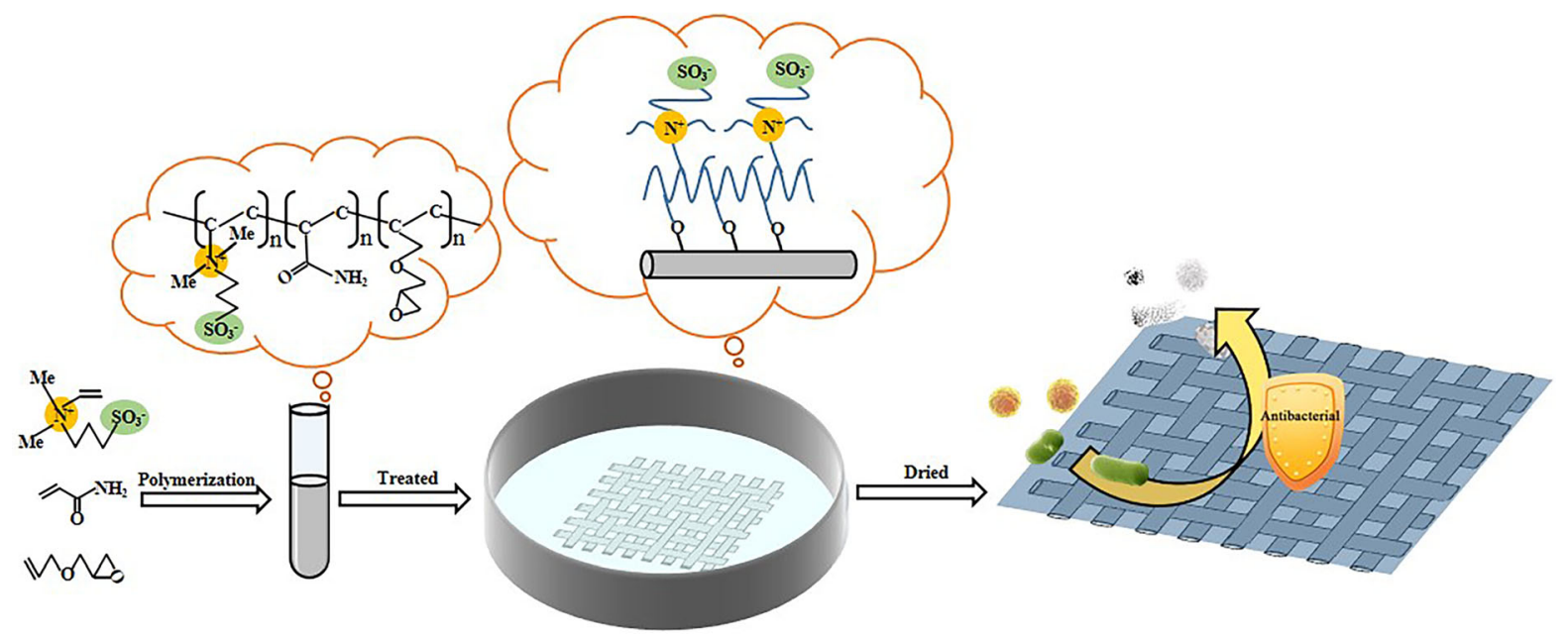

Scheme 1 Schematic illustration of the PSPB-AM-AGE grafting on cotton fabrics

\section{Antibacterial assay}

The antibacterial properties of PSPB-AM-AGE treated cotton textiles against $S$. aureus (ATCC6538) and E. coli (ATCC8739) were quantitatively evaluated by viable cell count method. Briefly, two samples $(5 \times 5 \mathrm{~cm}, 2$ pieces) of the fabrics (pristine cotton as the control, PSPB-AM-AGE treated cotton) were individually put into flasks. A $70 \mathrm{~mL}$ aliquot of PBS containing around $1 \times 10^{8} \mathrm{CFU} / \mathrm{mL}$ of bacteria was added to each of the two flasks. Once incubated, two flasks were shaken at the speed of $150 \mathrm{rpm}$ for $18 \mathrm{~h}$. Then, each solution of the flasks was 1:10 serially diluted $(400 \mu \mathrm{L}$ in $3.6 \mathrm{~mL}$ aliquot of PBS) five times, and $10 \mu \mathrm{L}$ from the undiluted and diluted solution were placed in columns on gridded six column square plates respectively (Dong 2018; Wang 2018). The agar plates were incubated at $37{ }^{\circ} \mathrm{C}$ for $18-24 \mathrm{~h}$. The survival rate was calculated by the ratio of bacterial colonies in PSPB-AM-AGE treated plates versus that of the pristine control. The antibacterial rate of textiles was calculated using the following equation.

Antibacterial rate $=\frac{N_{0}-N_{1}}{N_{0}}$

where $\mathrm{N}_{0}$ means the number of bacterial colonies on the pristine cotton fabrics after $16 \mathrm{~h}$ contact and $\mathrm{N}_{1}$ refers to that of treated cotton fabrics. According to GB/T 8629-2001 standard method, the durability of the PSPB-AM-AGE treated textiles against repeated launderings was investigated by the washing process
(Hassan 2017). Then the antibacterial activities of textiles were tested by aforementioned viable cell count method.

\section{Comprehensive performance evaluation}

In order to evaluate the influence of PSPB-AM-AGE finishing, the mechanical property, water absorption behavior, air/moisture permeability of fabric treated with PSPB-AM-AGE, and raw cotton are systematically studied.

Mechanical properties evaluation According to ISO9073-3, the mechanical performance of PSPBAM-AGE treated textiles was determined by YG026 strength tester (China) (Xu 2018). The pristine cotton was applied as a control sample. All samples were cut into $200 \times 5 \mathrm{~cm}$ to prepare for the experiment. The holding distance was $100 \mathrm{~mm}$ and the stretching velocity was $100 \mathrm{~mm} / \mathrm{min}$. Five repeats were measured for every kind of sample both in warp and weft. The breaking force and elongation through testing were obtained to appraise the mechanical property of PSPB-AM-AGE treated textiles and raw cotton, respectively.

Water absorption behavior The hydrophilicity of pristine cotton fabrics and PSPB-AM-AGE treated fabrics was tested by a DSA25 (KRUSS, German) dynamic contact angle measuring service and a wicking effect tester. Samples measuring $3 \times 15 \mathrm{~cm}$ 
were used and then immersed one side into an aqueous red ink solution. The height of the ink track was recorded to evaluate the water wicking ability of textiles (Chen 2016).

Moisture permeability Moisture permeability of all samples was determined by the change of weight before and after placing in a specialized instrument (YG601H-II, China) about $60 \mathrm{~min}$, where the temperature was $38 \pm 2{ }^{\circ} \mathrm{C}$ and relative humidity was $90 \pm 2 \%$.

Air permeability Through measurement under the pressure of $100 \mathrm{~Pa}$, air permeability of raw cotton textiles and PSPB-AM-AGE finished textiles was evaluated by YG461E-III fully automatu permeability instrument.

Whiteness performance According to the test method AATCC 110-2015 "Whiteness of Textile", Datacolor spectrophotometer was used to test the whiteness of the cotton fabrics before and after finishing with PSPB-AM-AGE.

\section{Bacterial attachment assay}

The pristine and treated fabrics of $5 \times 5 \mathrm{~cm}$ were washed with PBS twice, followed by sterilization under UV radiation for $40 \mathrm{~min}$. Thereafter, two samples were individually placed in flasks, which contains $30 \mathrm{~mL}$ bacterial suspension at a concentration of $10^{6} \mathrm{CFU} / \mathrm{mL}$, and incubated at $37^{\circ} \mathrm{C}$ for $6 \mathrm{~h}$. In order to observe under SEM, the samples were rinsed with PBS thrice and fixed with 3 vol \% glutaraldehyde in PBS overnight, and then subjected to serial dehydration with $20,30,40,50,60,70,80,90$ and $100 \%$ ethanol for $10 \mathrm{~min}$ respectively. Finally, the samples were dried at room temperature and observed under SEM (Li 2012).

\section{Cytotoxicity assay}

The viability of HaCat cells was used to evaluate the cytotoxicity performance of PSPB-AM-AGE. Samples were sterilized by UV irradiation. HaCat cells were grown in DMEM medium with $10 \%$ serum at $5 \%$ $\mathrm{CO}_{2}$ and $37{ }^{\circ} \mathrm{C}$. DMEM medium with $10 \%$ serum was used to make suspensions with different concentrations of $0 \mathrm{mg} / \mathrm{ml}, 0.5 \mathrm{mg} / \mathrm{ml}, 1.0 \mathrm{mg} / \mathrm{ml}, 2.0 \mathrm{mg} / \mathrm{ml}$,
$2.5 \mathrm{mg} / \mathrm{ml}$ of PSPB-AM-AGE. The cells in the logarithmic growth phase were seeded in 96-well plates at $4 \times 10^{3} /$ well and grown for $24 \mathrm{~h}$. Thereafter, the pristine medium was replaced by a medium with various concentrations of PSPB-AM-AGE prepared before. After $24 \mathrm{~h}$ of incubation, the medium was removed. We washed each well three times with PBS and added $100 \mu \mathrm{L}$ of medium containing $0.5 \mathrm{mg} / \mathrm{ml}$ MTT, which was incubated in a $37{ }^{\circ} \mathrm{C}$ incubator for $4 \mathrm{~h}$ at $5 \% \mathrm{CO}_{2}$. After discarding the medium, we added $100 \mu \mathrm{L}$ of deionized water to each well and measured the absorbance at $570 \mathrm{~nm}$ using a microplate reader. The relative activity of HaCat cells was calculated by the formula below.

Relative activity $=\frac{A_{0}-A_{1}}{A_{2}-A_{1}} \times 100$

where $A_{0}$ and $A_{2}$ are the absorbance of the cells incubated with PSPB-AM-AGE and control cells, $\mathrm{A}_{1}$ is the absorbance of background, which means the plate.

\section{Results and discussion}

Chemical structure of PSPB-AM-AGE

The chemical structure of SPB, PSPB-AM-AGE were obtained by Fourier-transform infrared spectroscopy (Fig. 1a). In PSPB-AM-AGE, the absorption peaks observed at 1203 and $1040 \mathrm{~cm}^{-1}$ could be attributed to the stretching vibration of the $\mathrm{SO}_{3}{ }^{-}$group. The further presence of the peaks at 605 and $528 \mathrm{~cm}^{-1}$ also showed the existence of the $\mathrm{SO}_{3}{ }^{-}$group. There were two peaks at 1483 and $1416 \mathrm{~cm}^{-1}$, which were assigned to the methyl group of quaternary ammonium salts $\left(\mathrm{N}^{+}-\mathrm{CH}_{3}\right)$ and $\mathrm{C}-\mathrm{N}$ (Chen 2011a). In contrast to the SPB spectra that showed the peaks at 1483 and $1416 \mathrm{~cm}^{-1}$, this implied that the polymerization of SPB was achieved. Furthermore, peaks at $3402 \mathrm{~cm}^{-1}$ and $3194 \mathrm{~cm}^{-1}$ confirmed the existence of $-\mathrm{NH}_{2}$ group. The strong absorption peaks at $1665 \mathrm{~cm}^{-1}$ and $1647 \mathrm{~cm}^{-1}$ could be attributed to the $\mathrm{C}=\mathrm{O}$ group of AM. However, due to the low concentration of AGE in the polymer, its characteristic absorption peak was not very transparent.

${ }^{1} \mathrm{H}-\mathrm{NMR}$ spectra of PSPB-AM-AGE was applied to get further information about the polymerization of SPB, AM, and AGE. The chemical structure of PSPB- 

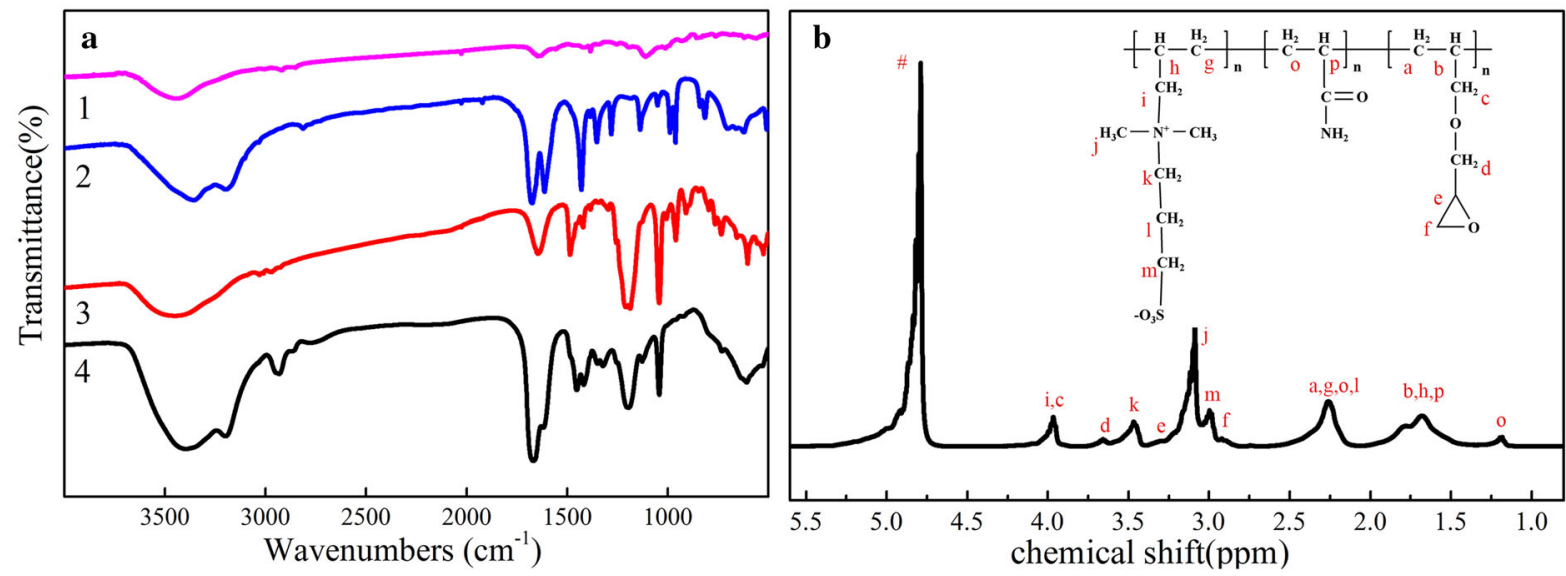

Fig. 1 Characterization of PSPB-AM-AGE: a FTIR spectra of the 1 AGE, 2 AM, 3 SPB and 4 PSPB-AM-AGE; $\mathbf{b}{ }^{1} \mathrm{H}-\mathrm{NMR}$ spectra of the PSPB-AM-AGE

AM-AGE was characterized by $500 \mathrm{MHz}{ }^{1} \mathrm{H}$ NMR spectra using $\mathrm{D}_{2} \mathrm{O}$ as a solvent. The typical ${ }^{1} \mathrm{H}-\mathrm{NMR}$ spectra for the prepared sulfobetaine copolymers with epoxy groups are shown in Fig. 1b, indicating the copolymers obtained.

To obtain the structure of the PSPB-AM-AGE, we measured the average molecular weight of polymers with GPC. Mw and Mn of polymers are $238 \mathrm{kDa}$ and $52 \mathrm{kDa}$, respectively. Thus $\mathrm{Mw} / \mathrm{Mn}$ of PSPB-AMAGE is around 4.5, indicating that the polymers obtained show a wide range of the molecular weight. Besides, the conversion yield of products was $80.2 \%$, which was calculated by polymer weighting.

\section{Characterization of treated cotton textiles}

\section{ATR-FTIR spectroscopic characterization}

FTIR spectra were acquired to gain evidence for the presence of PSPB-AM-AGE on the treated cotton (Fig. 2). Compared with pristine cotton, PSPB-AMAGE treated cotton exhibited three new absorption peaks at 1665,1540 , and $1206 \mathrm{~cm}^{-1}$ assigned in Fig. 2a, which were ascribed to $\mathrm{C}=\mathrm{O}, \mathrm{N}-\mathrm{H}$, and $\mathrm{SO}_{3}{ }^{-}$, indicating the presence of PSPB-AM-AGE on the treated cotton fabrics. Meanwhile, the band at 955 and $918 \mathrm{~cm}^{-1}$ (the absorption peaks of epoxy groups) was disappeared at the FTIR spectra of Fig. 2b, indicating the bonding reaction between epoxy groups at PSPB-AM-AGE and hydroxyl groups on cotton fabrics.
XPS spectra were employed to further characterize the PSPS-AM-AGE modified onto the surface of cotton fabric. As shown in Fig. 3, the peak observed at $168 \mathrm{eV}$ in the PSPB-AM-AGE as well as the finished cotton fabrics could be attributed to $S 2 p$, indicating the presence of $\mathrm{SO}_{3}{ }^{-}$group in those materials. Compared to the above two peaks, no obvious peak was found at such a range of spectra in the pristine textiles, confirming that it was the finishing of cotton with PSPB-AM-AGE that made fabrics with abundance of $\mathrm{SO}_{3}{ }^{-}$group. In addition, the PSPB-AM-AGE and PSPB-AM-AGE treated cotton both presented a peak at $402 \mathrm{eV}$ due to the positively charged nitrogen $\left(\mathrm{N}^{+}\right)$ of betaine monomer (Chen 2016), while there was no such peak in the samples of cotton fabrics as presented in Fig. 3. The change in elements was readily be quantified, thus covalently grafting of PSPB-AMAGE onto cotton fabrics was successful. Furthermore, In the high-resolution of PSPB-AM-AGE, the experimental molar ratio of $\mathrm{S}$ and $\mathrm{N}^{+}$was $0.9: 1.0$ as expected, which was calculated by intensity of $\mathrm{S} 2 \mathrm{p}$ and $\mathrm{N}^{+}$peaks. Meanwhile, the ratio of $\mathrm{S} 2 \mathrm{p} / \mathrm{N}^{+}$in the PSPB-AM-AGE treated cotton is 1.0:1.0, which was agree with theoretical values (the calculation was shown in the Supporting Materials, Fig. S1 and Tab. $\mathrm{S} 1)$. Overall, the peaks of N1s and S2p in the PSPBAM-AGE were stronger than those of in its treated cotton. The XPS characterization showed that the strategy proposed in this paper was practicable since the PSPB-AM-AGE was embedded in cellulose fibers. 

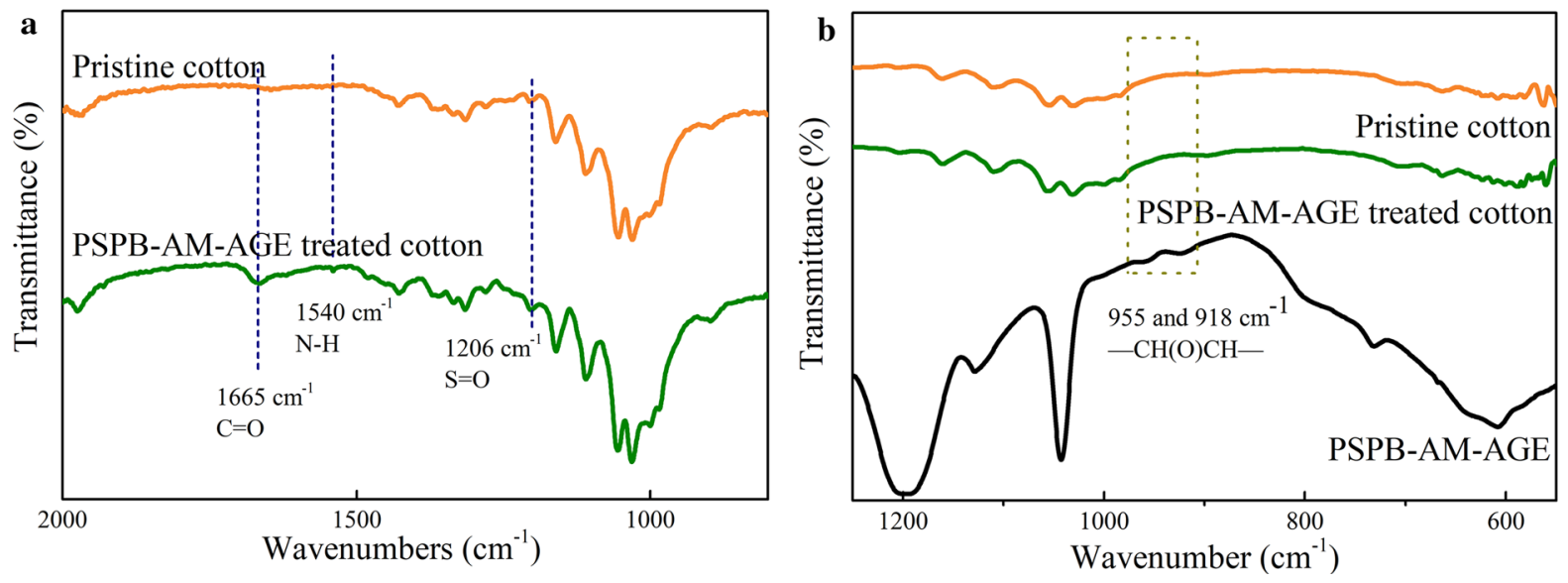

Fig. 2 FTIR spectra of the PSPB-AM-AGE, pristine and PSPB-AM-AGE treated cotton fabrics
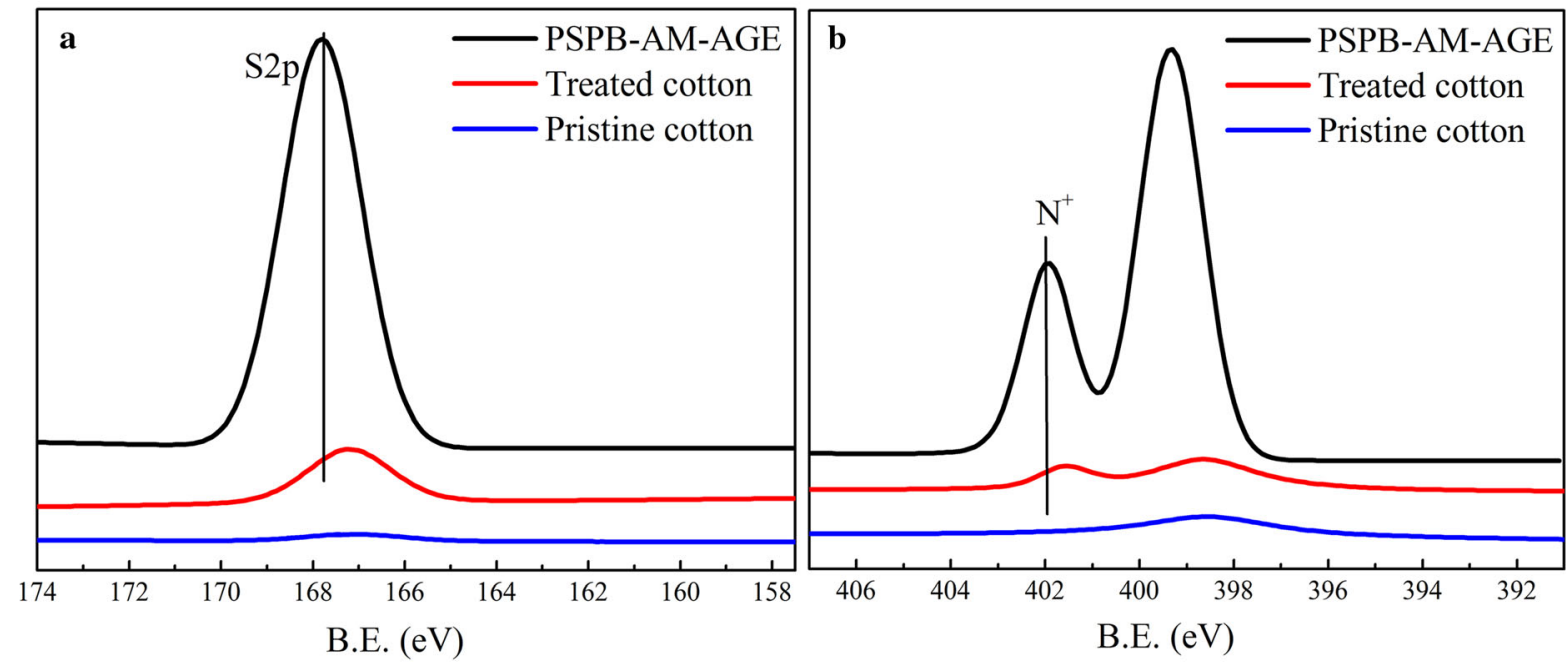

Fig. 3 XPS spectra of PSPB-AM-AGE, treated cotton and pristine cotton fabrics

\section{Morphology observation}

The morphologies of the PSPB-AM-AGE treated cotton and pristine cotton surfaces were obtained by SEM analysis. According to Fig. 4a, the pristine cotton surface was smooth and presented a grain, ravines, and distortion structure (Xu 2017). After being treated by PSPB-AM-AGE in Fig. 4b, compared to pristine cotton, the fabrics had a slight change in the surface covered with continuous films, which may due to the attachment of antibacterial agents (Fei 2018). Moreover, the rough presence of treated fabrics after washing 30 times from Fig. 4c confirmed it was robust against laundering. It is noticed that the process of PSPB-AM-AGE finishing cotton is carried out in the aqueous solvent. Thus, this confirmed that PSPBAM-AGE as the AFA is promising for large-scale manufacturing of antibacterial fabrics.

\section{Antibacterial properties of PSPB-AM-AGE treated cotton}

In order to investigate the antibacterial effect of PSPBAM-AGE treated cotton, we measured its antibacterial abilities (Chen 2016). Figure 5 presents the results of $S$. aureus as Gram-positive bacteria and E. coli as Gram-negative bacteria living on the culture flaks. The 

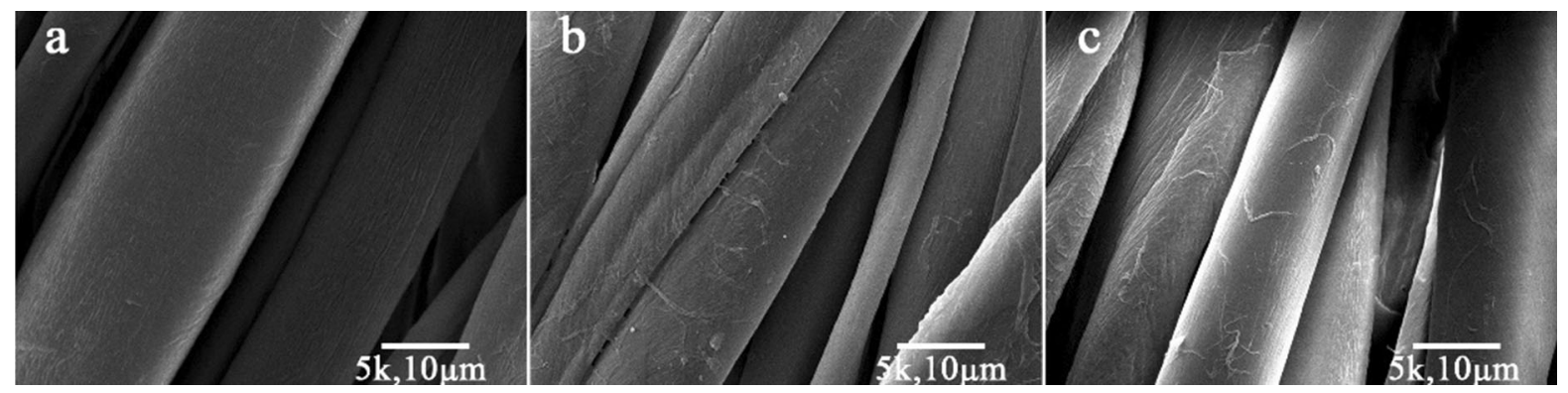

Fig. 4 SEM picture of a pristine cotton b PSPB-AM-AGE treated fabrics $\mathbf{c}$ fabrics treated with PSPB-AM-AGE after 30 laundering times

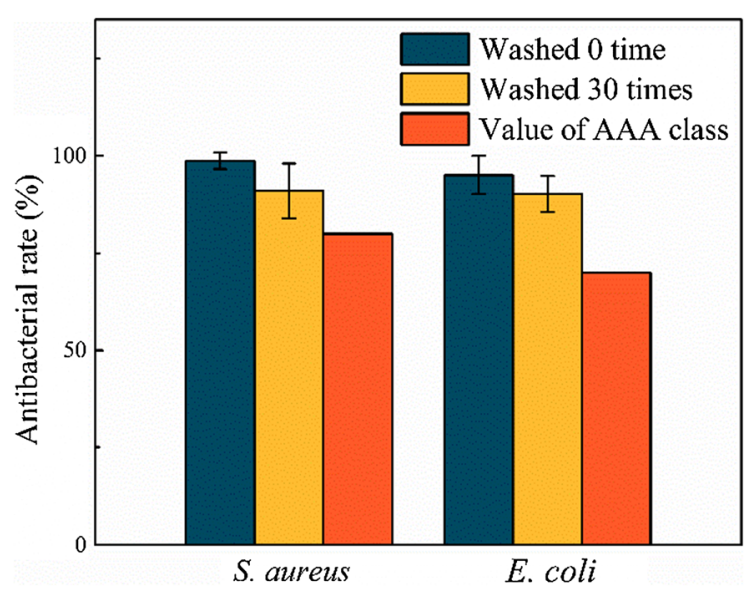

Fig. 5 Antibacterial activities against S. aureus and E. coli of cotton fabrics treated with PSPB-AM-AGE after 30 laundering times

number of survived colonies was recorded to evaluate the antimicrobial rate of treated cotton textiles. An additional experiment was carried to test if the AFA could leach from finished fabrics (Fig. S1, supporting information). As expected, both bacterial count against $S$. aureus and those to E. coli in the samples treated with PSPB-AM-AGE decreased significantly in contrast to pristine cotton textiles from Fig. 5. It can be calculated that the antibacterial rate of samples treated with PSPB-AM-AGE against $S$. aureus and E. coli are $98.74 \%$ and $95.18 \%$, separately, which are higher than the three-A criteria level of antibacterial fabrics that perform $80 \%$ and $70 \%$ to S. aureus and E. coli. This could be attributed to the broad-spectrum bacteriostatic and lethal effects of SPB, as derivatives of betaine with capacity to be amassed intracellularly at molar levels from extremely dilute Solutions (Cosquer 2004), which may inhibit the growth of microbes. Previous studies have established that this amphiphilic monomer can be used for polymerization (Zhou 2018). The proposed reaction mechanism of the grafting was that the epoxy groups of the polymer chains and the hydroxyl groups on cotton surface formed a covalent bond, thus obtaining functionalized cotton textiles as shown in scheme 1. The antibacterial mechanism of PSPB-AM-AGE could be concluded as (1) increasing diffusion through the bacterial cell wall with the long carbon chain of polybetaine (Ramadan 2018; Rojas 2018); (2) binding to single regulators on the bacterial membrane, indirectly interfering the biosynthesis of big molecular (Chen 2016; Chou 2016); (3) the presence of sulfonic acid groups along the polymer chain, the aqueous medium in microbes and/or their suspensions simultaneously promoting polymer hydration and reducing the $\mathrm{pH}$. A sudden change in $\mathrm{pH}$, however, promotes stress on the outer membrane and, if sufficiently drastic, destroys the membrane, resulting in enzyme damage, protein denaturation, and microbe death (Peddinti 2019).

To investigate the durable antimicrobial activities of cotton treated with PSPB-AM-AGE after laundering, the survival of colonies was recorded of original cotton and PSPB-AM-AGE finished cotton fabrics after 6 times washing, equivalently to 30 times of commercial washing. As shown in Fig. 6, the antibacterial rate of PSPB-AM-AGE finished cotton fabrics against $E$. coli is $90.24 \%$ and that of against $S$. aureus is $91.04 \%$, which indicate that cotton textiles grafted with PSPB-AM-AGE have durable antibacterial property. As for the leaching experiment of antibacterial agents from finished fabrics, the results showed that PSPB-AM-AGE was steadily grafted onto cotton textiles since there was no inhibition zone according to Fig. S2. 


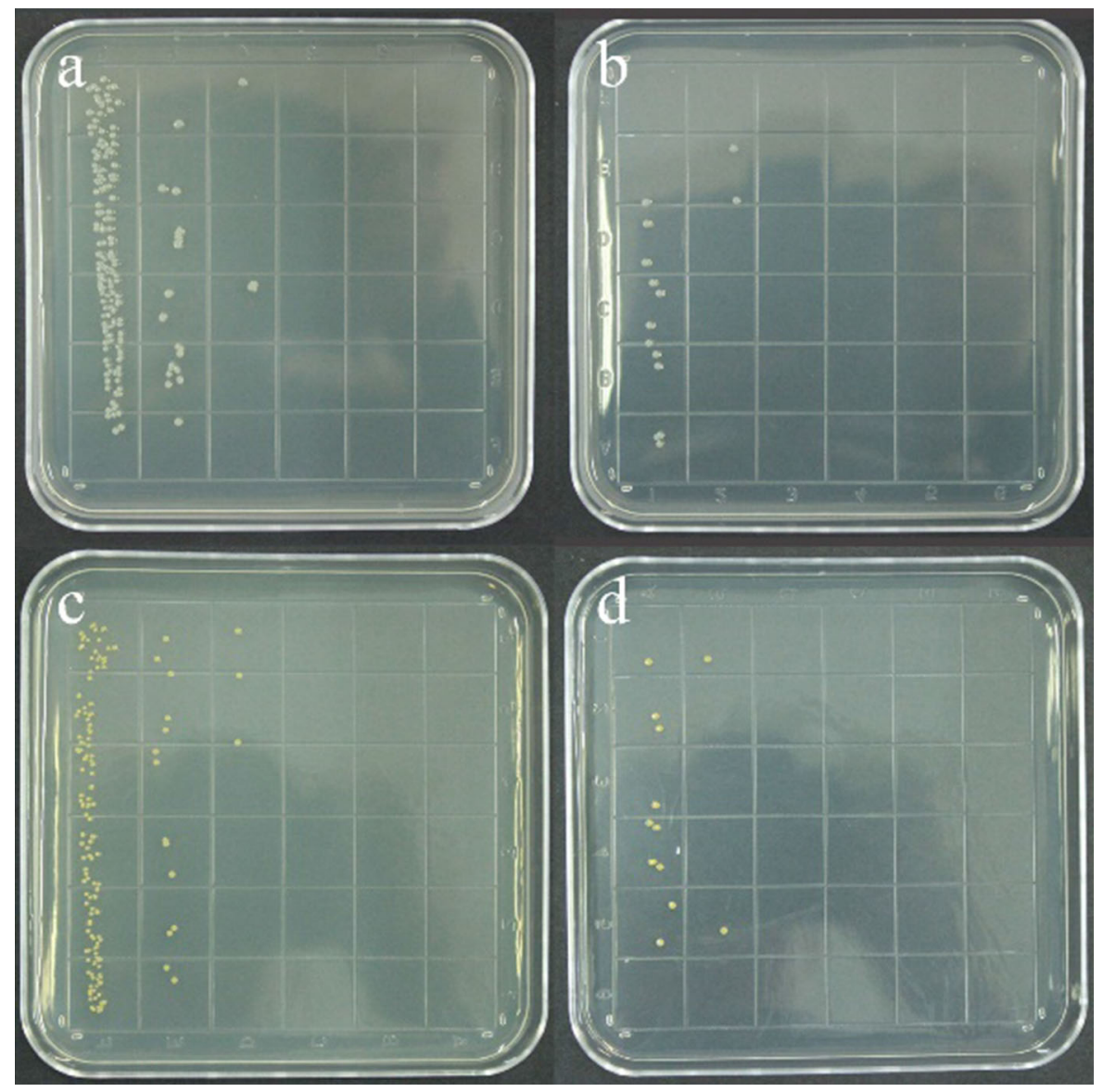

Fig. 6 Durable antimicrobial activities of cotton a pristine cotton against $E$. coli $\mathbf{b}$ PSPB-AM-AGE treated fabrics against $E$. coli after washing 30 times c pristine cotton against $S$. aureus (b) PSPB-AM-AGE treated fabrics against $S$. aureus after washing 30 times

\section{Antibacterial attachment of cotton finished with PSPB-AM-AGE}

Bacterial attachment on surfaces is generally followed by the growth of bacterial and the formation of biofilm, which could protect the bacterial from the impact of microbiocidal agents (Rojas 2018). Polysulfobetaine polymer, which contains an equimolar number of cationic and anionic groups, shows excellent inhibition to bacterial adhesion and the formation of biofilms in previous studies (Li 2012). However, reports on surface modification of textiles with polysulfobetaine for antibacterial attachment property are lacking. In this work, we select $S$. aureus and E. coli to investigate the resistance effects to bacterial adhesion of PSPB-AM-AGE grafted cotton fabrics. The results of bacterial adhesion after long-term incubation can be seen in Fig. 7, which shows that $S$. aureus and E. coli adhere readily to cotton fabrics. After treated with hydrophilic PSPB-AM-AGE, the number of adherent bacterial reduced obviously, which the layers with different thicknesses of the polymer on the fabrics had little effect on its antibacterial property. These results demonstrate that PSPBAM-AGE modified surface of textiles has a great performance to resistant the colonization of microbes, which is consistent with the antibacterial adhere property of zwitterionic polymers in the literature (Wang 2015). Since the zwitterionic polymers, carrying a positive and a negative charge on the same monomer unit, may cause a hydration layer near the surface, they could form a physical and energy barrier to prevent bacterial adhesion from the surface (Chen 2010). 


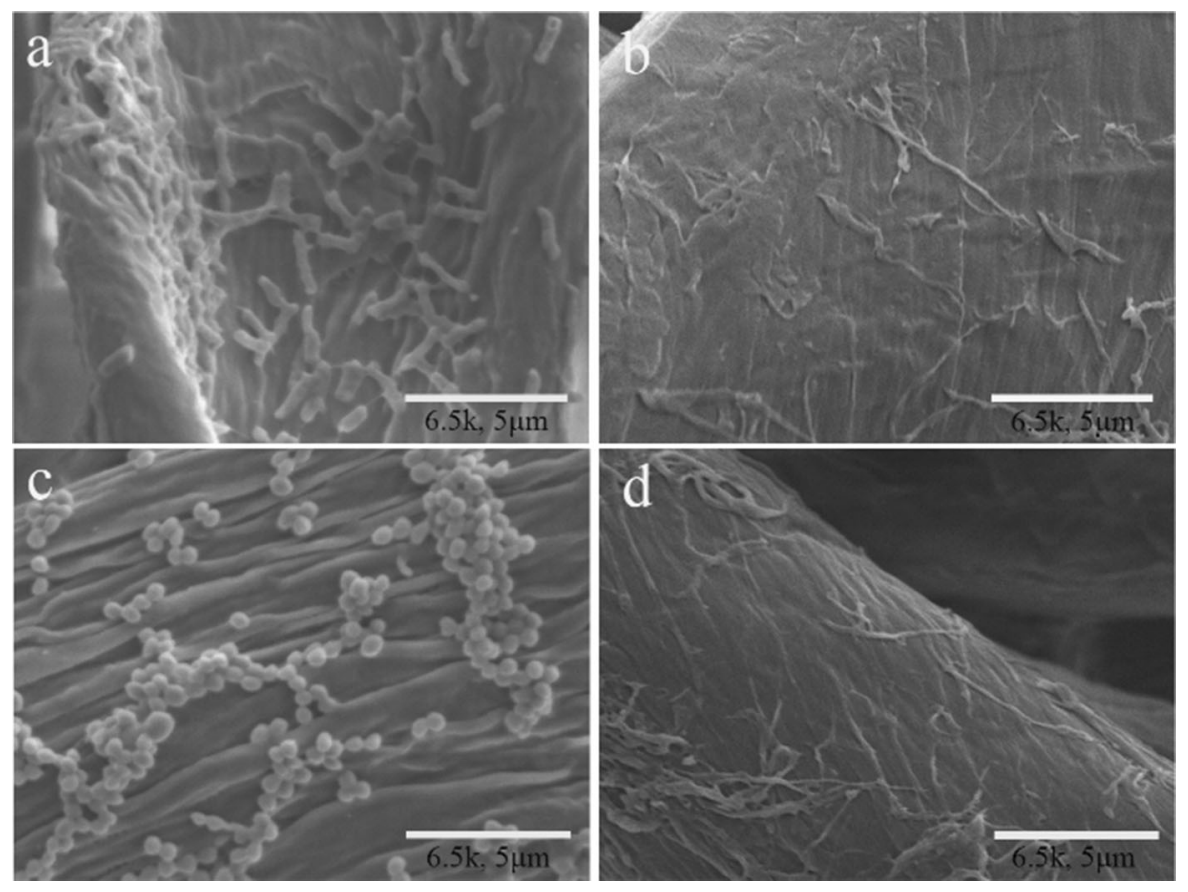

Fig. 7 SEM images of bacterial attachment on (a), (c) pristine cotton and (b), (d) PSPB-AM-AGE treated cotton fabrics after $6 \mathrm{~h}$ incubation. (a), (b) E. coli (ATCC8739); (c), (d) S. aureus (ATCC6538)

Hydrophilicity of cotton finished with PSPB-AMAGE

Capillary penetration and contact angle were used to investigate the hydrophilicity performance of cotton fabrics treated with PSPB-AM-AGE. As shown in Fig. 8 the overall wicking height of PSPB-AM-AGE treated cotton is obviously higher than that of pristine fabrics. The wicking height of treated cotton increased from $0 \mathrm{~cm}$ to $17.0 \mathrm{~cm}$ over a $45 \mathrm{~min}$ experiment, rising gently to the peak at $19.5 \mathrm{~cm}$ at the end.

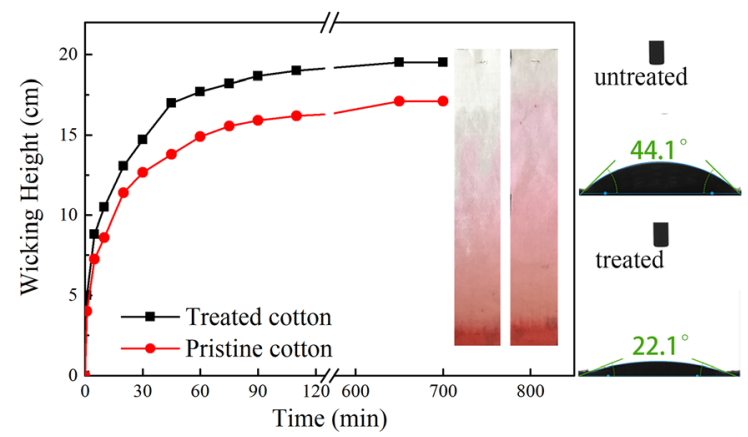

Fig. 8 Hydrophilicity performance of pristine cotton and PSPB-AM-AGE treated cotton fabrics
However, the wicking height of pristine cotton significantly rose to $12.7 \mathrm{~cm}$ after a $25 \mathrm{~min}$ experiment and then gradually hit the summit at $17.1 \mathrm{~cm}$ within $650 \mathrm{~min}$. From Fig. 8, the water contact angle of treated cotton decreased to $21.1^{\circ}$ over $5 \mathrm{~s}$, while that of raw cotton fabrics is $44.4^{\circ}$. These results show that the PSPB-AM-AGE treated cotton has better hydrophilicity than original materials, which attribute to the hydrophilic property of groups such as $-\mathrm{SO}_{3}$, $\mathrm{NH}_{2}$ on PSPB-AM-AGE agents.

\section{Mechanical performance of cotton finished with PSPB-AM-AGE}

The mechanical properties of fabrics treated with PSPB-AM-AGE and raw cotton were evaluated by breaking elongation and strength as shown in Fig. 9, which are critical elements for the application of textiles. The breaking strength of pristine textiles dropped from 723 to $669 \mathrm{~N}$ in the warp direction and from 393 to $363 \mathrm{~N}$ in the weft direction after finishing with the antibacterial agents PSPB-AM-AGE through pad-dry process. Meanwhile, it is found that the breaking elongation grew from $10.88 \%$ to $12.25 \%$ in 


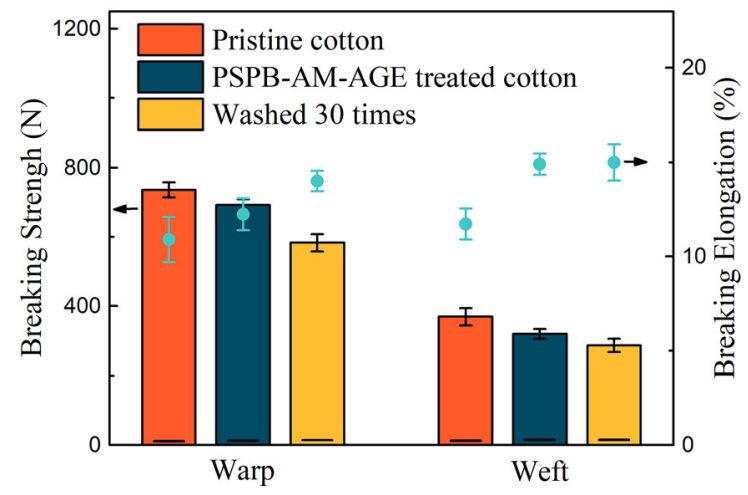

Fig. 9 Mechanical performance including breaking strength and breaking elongation of pristine cotton, PSPB-AM-AGE treated cotton and fabrics treated with PSPB-AM-AGE after 30 laundering times

the warp direction (increased by 12.59\%) and increase from $11.72 \%$ to $14.90 \%$ in the weft direction (a rose by $27.13 \%$ ). The durability of mechanical properties after 30 wash cycles was checked and it was revealed that the tensile performance of PSPB-AM-AGE treated cotton showed a reduce of around $15 \%$. These results show that essential cotton properties can be largely preserved in the treatment process. It is noted that we prepared AFA by polymerization (PSPB) of SPB and maleic anhydride and apply for the treatment of cotton textiles in our previous work, resulting in the breaking strength retention after finishing is $91.93 \%$ (Zhou 2018). However, the mechanical performance of cotton fabrics treated with PSPB-AM-AGE in this work was improved than PSPB finished cotton textiles, which may attribute to the reason, that AGE could link the antibacterial agents with cotton fabrics leading to less damage to cellulose fibers than that of maleic acid.

Air permeability and moisture penetrability of cotton finished with PSPB-AM-AGE

The transmission of vapor and moisture is an important factor to textiles for the wearing comfort of the human body (Xu 2018). Thus, air permeability and moisture permeability were applied. In contrast to original cotton fabrics, PSPB-AM-AGE modified cotton fabrics had a slight reduction of air permeability that fell from $72.08 \mathrm{~mm} / \mathrm{s}$ to $62.11 \mathrm{~mm} / \mathrm{s}$, with the retention of $86.16 \%$ from Fig. 10a. The air permeability drop is in agreement with the literature $(\mathrm{Xu}$
2018). In addition, compared to the air permeability of fabrics treated with short chain betaine in the literature (dropped by 21.3\%) (He 2016), that of treated with PSPB-AM-AGE showed no obvious decline. While the moisture permeability experienced an increase from $6665.73 \mathrm{~m}^{2} \cdot 24 \mathrm{~h}$ to $6818.38 \mathrm{~m}^{2} \cdot 24 \mathrm{~h}$ after treated (grew by $2.16 \%$ ) as shown in Fig. 10b, which demonstrated that PSPB-AM-AGE modified cotton fabrics had a better moisture transmission due to the improved hydrophilicity of the fabrics.

Whiteness performance of cotton finished with PSPB$A M-A G E$

To analyze whether the antibacterial agent can affect the appearance of cotton fabrics, the whiteness of fabrics finished with PSPB-AM-AGE was detected by the Datacolor spectrophotometer. The results were shown in Tab. 1. The whiteness of cotton fabrics dropped from 73.2 to 68.7 after finishing with PSPBAM-AGE as the high curing temperature and low $\mathrm{pH}$ of antibacterial agents. Compared to the whiteness reduction of fabrics treated with chitosan and nanosilver particles (Xu 2018), the retention of cotton pristine appearance treated with PSPB-AM-AGE is better.

The cytotoxicity of PSPB-AM-AGE was applied to evaluate effects on human skin HaCat cells using different concentrations of PSPB-AM-AGE. We used classic MTT methods to achieve the goal. The results showed in Fig. 11. Overall, the relative activity of HaCat cells was affected by PSPB-AM-AGE additives. When the concentration of PSPB-AM-AGE got to $2 \mathrm{mg} / \mathrm{mL}$, the cell viability was ca. $97.6 \%$. With more additions of PSPB-AM-AGE, the activity of cells declined obviously. However, the cell viability was ca. $84.8 \%$, still over $80 \%$, when the PSPB-AMAGE concentration was $2.5 \mathrm{mg} / \mathrm{mL}$. These results demonstrated that PSPB-AM-AGE had no apparent cytotoxicity to human skin cells, which means the metabolism activity of that cell hardly be affected by antibacterial agents even at high concentrations.

\section{Conclusions}

A novel zwitterionic sulfobetaine polymer of PSPBAM-AGE with antibacterial function was demonstrated in this study. An ideal surface model of antibacterial cotton was covalently grafted with 

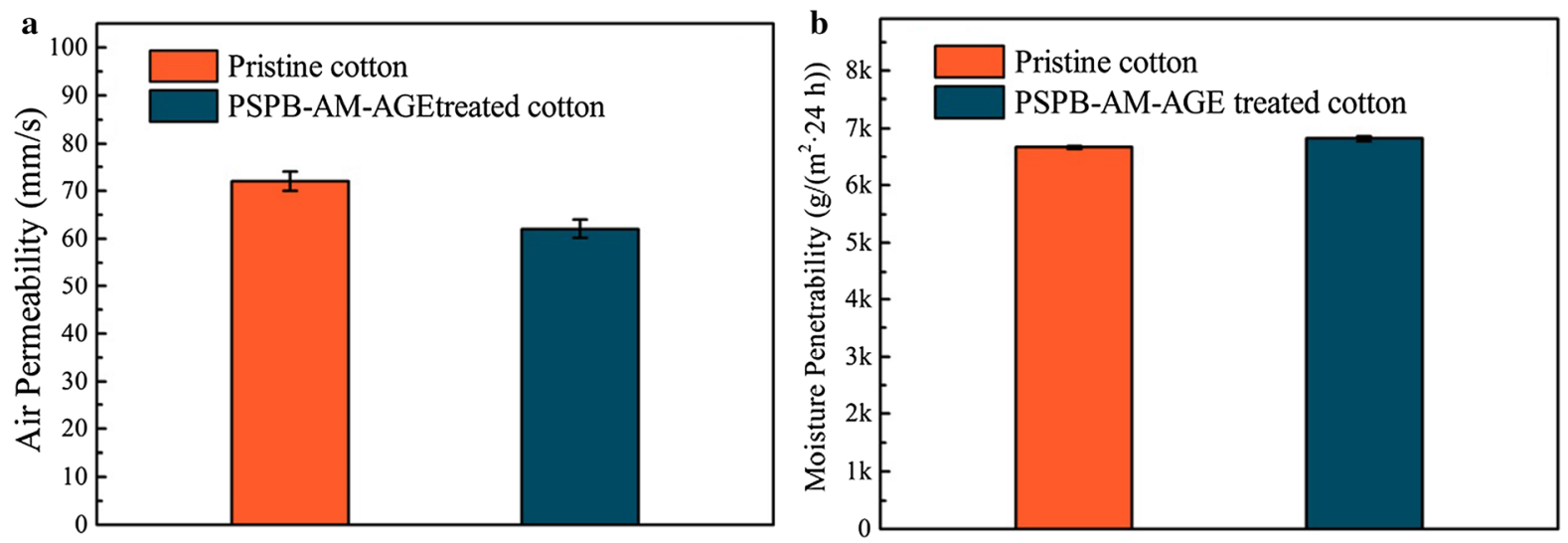

Fig. 10 Air and moisture penetrability of pristine cotton and PSPB-AM-AGE treated cotton fabrics

Tab. 1 Whiteness performance of pristine cotton and PSPB-AM-AGE treated cotton fabrics

\begin{tabular}{llr}
\hline Samples & Whiteness/\% & Change of whiteness \\
\hline Pristine cotton & 73.2 & -4.5 \\
PSPB-AM-AGE treated cotton & 68.7 & \\
\hline
\end{tabular}

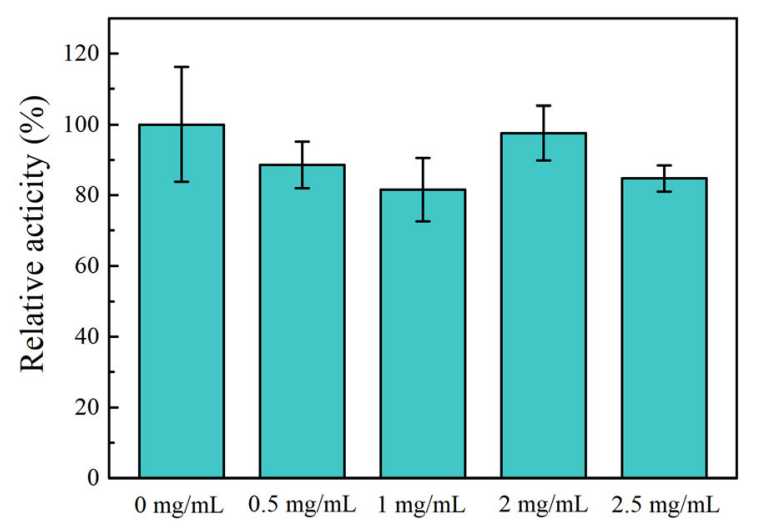

Concentration of PSPB-AM-AGE

Fig. 11 Cytotoxicity of PSPB-AM-AGE to HaCat cells at various concentrations after $24 \mathrm{~h}$ incubation

PSPB-AM-AGE via a ring opening reaction. The FTIR, XPS, and SEM results showed that PSPB-AMAGE was successfully grafted onto cotton fabrics. The viable cell count test proved a significant decrease of bacterial colonies in comparison to the pristine cotton surface, with antibacterial rate against $E$. coli and $S$. aureus reaching $95.18 \%$ and $98.74 \%$, separately. Cotton textiles treated with PSPB-AM-AGE had a durable antibacterial property, which exhibited over
90\% against both E. coli and S. aureus and no leaching from substrates. The mechanical properties of cotton were slightly dropped in the treatment process, though improved a lot compared to the previous PSPB finished cotton. Furthermore, the wearing comfort performance of cotton namely hydrophilicity, air, and water permeability were improved after being finished with PSPB-AM-AGE. The treatment of cotton fabrics had no obvious effects on its appearance. Furthermore, PSPB-AM-AGE demonstrated no apparent cytotoxicity to human skin cells. The synthesis of polysulfobetaine like PSPB-AM-AGE shows a potential application on the next generation of antibacterial cotton fabrics.

Acknowledgments We thank the National Natural Science Foundation of China (No.52003108), and the financial support from the Nation Key R\&D Program of China (2017YFB0309100), and the Key Laboratory of Eco-textiles, Ministry of Education-(supported by "the Fundamental Research Funds for the Central Universities NO. JUSRP52007A).

\section{References}

Chang Y, Chang WJ, Shih YJ et al (2011) Zwitterionic sulfobetaine-grafted poly(vinylidene fluoride) membrane with 
highly effective blood compatibility via atmospheric plasma-induced surface copolymerization. ACS Appl Mater Interfaces 3(4):1228-1237

Chen S, Chen S, Jiang S et al (2011a) Environmentally friendly antibacterial cotton textiles finished with siloxane sulfopropylbetaine. ACS Appl Mater Interfaces 3(4):1154-62

Chen S, Chen S, Jiang S et al (2011b) Synthesis and characterization of siloxane sulfobetaine antimicrobial agents. Surf Sci 605(11-12):L25-L28

Chen S, Li L, Zhao C, Zheng J (2010) Surface hydration: principles and applications toward low-fouling/nonfouling biomaterials. Polymer 51(23):5283-5293

Chen S, Yuan L, Li Q et al (2016) Durable antibacterial and nonfouling cotton textiles with enhanced comfort via zwitterionic sulfopropylbetaine coating. Small 12(26):3516-21

Chou YN, Wen TC, Chang Y (2016) Zwitterionic surface grafting of epoxylated sulfobetaine copolymers for the development of stealth biomaterial interfaces. Acta Biomater 40:78-91

Cosquer A, Ficamos M, Jebbar M et al (2004) Antibacterial activity of glycine betaine analogues: involvement of osmoporters. Bioorg Med Chem Lett 14(9):2061-2065

Dann AB, Hontela A (2011) Triclosan: environmental exposure, toxicity and mechanisms of action. J Appl Toxicol 31(4):285-311

Ding X, Duan S, Ding X et al (2018) Versatile antibacterial materials: an emerging arsenal for combatting bacterial pathogens. Adv Func Mater 28(40): 1802140

Dong J, Ghiladi RA, Wang Q et al (2018) Protoporphyrin IX conjugated bacterial cellulose via diamide spacer arms with specific antibacterial photodynamic inactivation against Escherichia coli. Cellulose 25(3):1673-1686

El-Nahhal IM, Salem J, Anbar R et al (2020) Preparation and antimicrobial activity of ZnO-NPs coated cotton/starch and their functionalized $\mathrm{ZnO}-\mathrm{Ag} /$ cotton and $\mathrm{Zn}$ (II) curcumin/cotton materials. Sci Rep 10(1):5410

Fei Z, Liu B, Zhu M et al (2018) Antibacterial finishing of cotton fabrics based on thiol-maleimide click chemistry. Cellulose 25(5):3179-3188

Ganewatta MS, Tang C (2015) Controlling macromolecular structures towards effective antimicrobial polymers. Polymer 63:A1-A29

Gao D, Li Y, Lyu B et al (2019) Construction of durable antibacterial and anti-mildew cotton fabric based on P(DMDAAC-AGE)/Ag/ZnO composites. Carbohyd Polym 204:161-169

Hanczvikkel A, Vig A, Toth A et al (2019) Survival capability of healthcare-associated, multidrug-resistant bacteria on untreated and on antimicrobial textiles. $\mathbf{J}$ Ind Tex 48(7):1113-1135

Hassan MS, Ali HE, Ali NM (2017) Antibacterial activity of cotton fabrics treated with poly (vinyl alcohol)/ZnO nanocomposites, photocatalyzed by UV Irradiation. J Vinyl Add Tech 23:E34-E39

He L, Gao C, Li S et al (2017) Non-leaching and durable antibacterial textiles finished with reactive zwitterionic sulfobetaine. J Ind Eng Chem 46:373-378

He L, Li S, Chung CT et al (2016) Constructing safe and durable antibacterial textile surfaces using a robust graft-to strategy via covalent bond formation. Sci Rep 6(1):36327
Li M, Neoh KG, Xu LQ et al (2012) Surface modification of silicone for biomedical applications requiring long-term antibacterial, antifouling, and hemocompatible properties. Langmuir 28(47):16408-16422

Li S, Lin X, Liu Y et al (2019) Phosphorus-nitrogen-siliconbased assembly multilayer coating for the preparation of flame retardant and antimicrobial cotton fabric. Cellulose 26(6):4213-4223

Lim SH, Hudson SM (2003) Review of chitosan and its derivatives as antimicrobial agents and their uses as textile chemicals. J Macromol Sci Polymer Rev 43(2):223-269

Liu Y, He Q, Li R et al (2017) Durable antimicrobial cotton fabrics treated with a novel N-halamine compound. Fibers Polym 17(12):2035-2040

Lu Z, Liu J, Dong C et al (2019) Durable multifunctional antibacterial and hydrophobic cotton fabrics modified with linear fluorinated pyridinium polysiloxane. Cellulose 26(12):7483-7494

Mitra D, Li M, Kang ET et al (2019) Transparent copper-based antibacterial coatings with enhanced efficacy against pseudomonas aeruginosa. ACS Appl Mater Interfaces 11(1):73-83

Peddinti BST, Scholle F, Vargas MG et al (2019) Inherently self-sterilizing charged multiblock polymers that kill drugresistant microbes in minutes. Mater Horiz 6(10):2056-2062

Ramadan DR, Elbardan AA, Bekhit AA et al (2018) Synthesis and characterization of novel dimeric s-triazine derivatives as potential anti-bacterial agents against MDR clinical isolates. New J Chem 42(13):10676-10688

Rehan M, El-Naggar ME, Mashaly HM, Wilken R (2018) Nanocomposites based on chitosan/silver/clay for durable multi-functional properties of cotton fabrics. Carbohydr Polym 182:29-41

Rojas ER, Billings G, Odermatt PD et al (2018) The outer membrane is an essential load-bearing element in Gramnegative bacteria. Nature 559(7715):617-621

U1 S, Islam B, B. S. (2020a) synergistic combination of shrimp shell derived chitosan polysaccharide with Citrus sinensis peel extract for the development of colourful and bioactive cellulosic textile. Int J Biol Macromol 158:94-103

Shen William, Zhang L, Li X et al (2019) Binary silanization and silver nanoparticle encapsulation to create superhydrophobic cotton fabrics with antimicrobial capability. Sci Rep 9(1):2045-2322

Si Y, Zhang Z, Wu W et al (2018) Daylight-driven rechargeable antibacterial and antiviral nanofibrous membranes for bioprotective applications. Sci Adv 4(3):5931

Sivashanmugan K, Liu PC, Tsai KW et al (2017) An antifouling nanoplasmonic SERS substrate for trapping and releasing a cationic fluorescent tag from human blood solution. Nanoscale 9(8):2865-2874

Sundaram HS, Han X, Nowinski AK et al (2014) Achieving onestep surface coating of highly hydrophilic poly(carboxybetaine methacrylate) polymers on hydrophobic and hydrophilic surfaces. Adv Mater Interfaces 1(6): 1400071

U1 Shahid, Islam Butola, B. S. (2020b) A synergistic combination of shrimp shell derived chitosan polysaccharide with Citrus sinensis peel extract for the development of colourful and bioactive cellulosic textile. International Journal of Biological Macromolecules 158:94-103 
Wang Q, Chen W, Zhang Q et al (2018) Preparation of photodynamic P(MMA-co-MAA) composite nanofibers doped with MMT: a facile method for increasing antimicrobial efficiency. Appl Surf Sci 457:247-255

Wang R, Neoh KG, Kang ET (2015) Integration of antifouling and bactericidal moieties for optimizing the efficacy of antibacterial coatings. J Colloid Interface Sci 438:138-148

Wang Y, Yin M, Li Z et al (2018) Preparation of antimicrobial and hemostatic cotton with modified mesoporous particles for biomedical applications. Colloids Surf B Biointerfaces 165:199-206

Ward M, Sanchez M, Elasri MO, Lowe AB (2006) Antimicrobial activity of statistical polymethacrylic sulfopropylbetaines against gram-positive and gram-negative bacteria. J Appl Polym Sci 101(2):1036-1041

Wieczorek D, Dobrowolski A, Staszak K et al (2017) Synthesis, surface and antimicrobial activity of piperidine-based sulfobetaines. J Surfactants Deterg 20(1):151-158

Wu G, Yang Q, Long M et al (2015) Evaluation of agar dilution and broth microdilution methods to determine the disinfectant susceptibility. J Antibiot 68(11):661-665
Xu L, Wang W, Yu D (2017) Preparation of a reactive flame retardant and its finishing on cotton fabrics based on click chemistry. RSC Adv 7(4):2044-2050

Xu Q, Ke X, Cai D et al (2018) Silver-based, single-sided antibacterial cotton fabrics with improved durability via an 1-cysteine binding effect. Cellulose 25(3):2129-2141

Zhang S, Yang X, Tang B et al (2018) New insights into synergistic antimicrobial and antifouling cotton fabrics via dually finished with quaternary ammonium salt and zwitterionic sulfobetaine. Chem Eng J 336:123-132

Zhang Z, Chen S, Chang Y, Jiang S (2006) Surface grafted sulfobetaine polymers via atom transfer radical polymerization as superlow fouling coatings. $J$ phys chem B 110(22):10799-10804

Zhou L, Wang H, Du J et al (2018) Eco-friendly and durable antibacterial cotton fabrics prepared with polysulfopropylbetaine. Fibers Polym 19(6):1228-1236

Publisher's Note Springer Nature remains neutral with regard to jurisdictional claims in published maps and institutional affiliations. 